PROCEEDINGS OF THE

AMERICAN MATHEMATICAL SOCIETY

Volume 128, Number 12, Pages 3595-3599

S 0002-9939(00)05552-0

Article electronically published on June 7, 2000

\title{
EQULIBRIUM MEASURE OF A PRODUCT SUBSET OF $\mathbb{C}^{n}$
}

\author{
ZBIGNIEW BŁOCKI
}

(Communicated by Steven R. Bell)

\begin{abstract}
In this note we show that an equilibrium measure of a product of two subsets of $\mathbb{C}^{n}$ and $\mathbb{C}^{m}$, respectively, is a product of their equilibrium measures. We also obtain a formula for $\left(d d^{c} \max \{u, v\}\right)^{p}$, where $u, v$ are locally bounded plurisubharmonic functions and $2 \leq p \leq n$.
\end{abstract}

\section{INTRODUCTION}

Let $E$ be a bounded subset of $\mathbb{C}^{n}$. The function

$$
V_{E}:=\sup \left\{u \in P S H\left(\mathbb{C}^{n}\right):\left.u\right|_{E} \leq 0, \sup _{z \in \mathbb{C}^{n}}\left(u(z)-\log ^{+}|z|\right)<\infty\right\}
$$

is called a global extremal function (or the Siciak extremal function) of E. It is known that $V_{E}^{*}$, the upper regularization of $V_{E}$, is plurisubharmonic in $\mathbb{C}^{n}$ if and only if $E$ is not pluripolar. In such a case, by [BT1], $\left(d d^{c} V_{E}^{*}\right)^{n}$ is a well defined nonnegative Borel measure and it is called an equilibrium measure of $E$. We refer to $\underline{\mathrm{Kl}}$ for a detailed exposition of this topic.

In this note we shall show

Theorem 1. Let $E$ and $F$ be nonpluripolar bounded subsets of $\mathbb{C}^{n}$ and $\mathbb{C}^{m}$, respectively. Then

$$
V_{E \times F}^{*}=\max \left\{V_{E}^{*}, V_{F}^{*}\right\}
$$

and

$$
\left(d d^{c} V_{E \times F}^{*}\right)^{n+m}=\left(d d^{c} V_{E}^{*}\right)^{n} \wedge\left(d d^{c} V_{F}^{*}\right)^{m} .
$$

Note that here we treat $V_{E}^{*}$ (resp. $V_{F}^{*}$ ) as a function of $\mathbb{C}^{n+m}$ independent of the last $m$ (respectively first $n$ ) variables.

The formula (1) was proved by Siciak (see [Si]) for $E, F$ compact (see also [Ze for a proof using the theory of the complex Monge-Ampère operator). For $n=m=1$ the proof of (2) can be found in [BT2].

If $E \subset D$, where $D$ is a bounded domain in $\mathbb{C}^{n}$, then the function

$$
u_{E, D}:=\sup \left\{v \in P S H(D): v \leq 0,\left.v\right|_{E} \leq-1\right\}
$$

is called a relative extremal function of $E$. Combining our methods of the proof of Theorem 1 with a result from [EP] we can also obtain

Received by the editors February 18, 1999.

2000 Mathematics Subject Classification. Primary 32U15; Secondary 32W20.

This work was partially supported by KBN Grant \#2 PO3A 00313.

(C)2000 American Mathematical Society 
Theorem 2. Let $D$ be a bounded domain in $\mathbb{C}^{n}$ and $G$ a bounded domain in $\mathbb{C}^{m}$. Then for arbitrary subsets $E \subset D, F \subset G$ we have

$$
u_{E \times F, D \times G}^{*}=\max \left\{u_{E, D}^{*}, u_{F, G}^{*}\right\}
$$

and

$$
\left(d d^{c} u_{E \times F, D \times G}^{*}\right)^{n+m}=\left(d d^{c} u_{E, D}^{*}\right)^{n} \wedge\left(d d^{c} u_{F, G}^{*}\right)^{m} .
$$

The relative Monge-Ampère capacity of $E \subset D$ is defined by

$$
c(E, D):=\sup \left\{\int_{E}\left(d d^{c} u\right)^{n}: u \in P S H(D),-1 \leq u \leq 0\right\},
$$

provided that $E$ is Borel. If $E \subset D$ is arbitrary, then, as usual, we can define

$$
\begin{aligned}
c^{*}(E, D) & :=\inf _{E \subset U, U \text { open }} c(U, D), \\
c_{*}(E, D) & :=\sup _{K \subset E, K \text { compact }} c(K, D) .
\end{aligned}
$$

By [BT1, if $E \Subset D$ and $D$ is hyperconvex (that is $\left(u_{E, D}\right)_{*}=0$ on $\partial D$ ), then

$$
c^{*}(E, D)=\int_{D}\left(d d^{c} u_{E, D}^{*}\right)^{n} .
$$

Moreover, $c^{*}(E, D)=c(E, D)=c_{*}(E, D)$ if $E$ is Borel. Theorem 2 thus gives

Theorem 3. Assume that $D$ and $G$ are bounded hyperconvex domains in $\mathbb{C}^{n}$ and $\mathbb{C}^{m}$, respectively. Then for $E \Subset D, F \Subset G$ we have

$$
c^{*}(E \times F, D \times G)=c^{*}(E, D) c^{*}(F, G) .
$$

I would like to thank N. Levenberg for inspiring discussions and E. Poletsky for his help in the proof of Lemma 8 below.

\section{ProOFs}

If $\Omega$ is an open subset of $\mathbb{C}^{n}$ and $1 \leq p \leq n$, then by [BT1] the mapping

$$
\left(u_{1}, \ldots, u_{p}\right) \longmapsto d d^{c} u_{1} \wedge \cdots \wedge d d^{c} u_{p}
$$

is well defined on the set $\left(P S H \cap L_{l o c}^{\infty}(\Omega)\right)^{p}$ and its values are nonnegative currents of bidegree $(p, p)$. Moreover, (4) is symmetric and continuous with respect to decreasing sequences. First, we shall prove

Theorem 4. Let $u, v$ be locally bounded plurisuharmonic functions. Then, if $2 \leq$ $p \leq n$, we have

$$
\begin{aligned}
& \left(d d^{c} \max \{u, v\}\right)^{p} \\
& \quad=d d^{c} \max \{u, v\} \wedge \sum_{k=0}^{p-1}\left(d d^{c} u\right)^{k} \wedge\left(d d^{c} v\right)^{p-1-k}-\sum_{k=1}^{p-1}\left(d d^{c} u\right)^{k} \wedge\left(d d^{c} v\right)^{p-k} .
\end{aligned}
$$

Proof. We leave it as an exercise to the reader to show that a simple inductive argument reduces the proof to the case $p=2$. By the continuity of (4) under decreasing sequences we may also assume that $u, v$ are smooth.

Let $\chi: \mathbb{R} \rightarrow[0,+\infty)$ be smooth and such that $\chi(x)=0$ if $x \leq-1, \chi(x)=x$ if $x \geq 1$ and $0 \leq \chi^{\prime} \leq 1, \chi^{\prime \prime} \geq 0$ everywhere. Define

$$
\psi_{j}:=v+\frac{1}{j} \chi(j(u-v)) .
$$


Denote for simplicity $w=\max \{u, v\}$ and $\alpha=u-v$. We can easily check that $\psi_{j} \downarrow w$ as $j \uparrow \infty$. An easy computation gives

$$
d d^{c}(\chi(j \alpha) / j)=\chi^{\prime}(j \alpha) d d^{c} \alpha+j \chi^{\prime \prime}(j \alpha) d \alpha \wedge d^{c} \alpha .
$$

Therefore

$$
d d^{c} \psi_{j}=\chi^{\prime}(j \alpha) d d^{c} u+\left(1-\chi^{\prime}(j \alpha)\right) d d^{c} v+j \chi^{\prime \prime}(j \alpha) d \alpha \wedge d^{c} \alpha
$$

and, in particular, $\psi_{j}$ is plurisubharmonic.

From the definition of $\psi_{j}$ we obtain

$$
\left(d d^{c} \psi_{j}\right)^{2}=\left(d d^{c} v\right)^{2}+2 d d^{c}(\chi(j \alpha) / j) \wedge d d^{c} v+\left(d d^{c}(\chi(j \alpha) / j)\right)^{2} .
$$

We have weak convergences

$$
\begin{aligned}
\left(d d^{c} \psi_{j}\right)^{2} & \longrightarrow\left(d d^{c} w\right)^{2}, \\
d d^{c}(\chi(j \alpha) / j) \wedge d d^{c} v & \longrightarrow d d^{c}(w-v) \wedge d d^{c} v,
\end{aligned}
$$

so it remains to analyze the third term of the right-hand side of (6). Using (5) and the fact that $\left(d \alpha \wedge d^{c} \alpha\right)^{2}=0$, we compute

$$
\begin{aligned}
\left(d d^{c}(\chi(j \alpha) / j)\right)^{2} & =\left(\chi^{\prime}(j \alpha)\right)^{2}\left(d d^{c} \alpha\right)^{2}+2 j \chi^{\prime}(j \alpha) \chi^{\prime \prime}(j \alpha) d \alpha \wedge d^{c} \alpha \wedge d d^{c} \alpha \\
& =d\left[\left(\chi^{\prime}(j \alpha)\right)^{2} d^{c} \alpha \wedge d d^{c} \alpha\right] \\
& =d d^{c}(\gamma(j \alpha) / j) \wedge d d^{c} \alpha,
\end{aligned}
$$

where $\gamma: \mathbb{R} \rightarrow \mathbb{R}$ is such that $\gamma^{\prime}=\left(\chi^{\prime}\right)^{2}$. In fact, if $\gamma$ is chosen so that $\gamma(-1)=0$, then $\gamma(j x) / j \downarrow \max \{0, x\}$ as $j \uparrow \infty$ and

$$
\left(d d^{c}(\chi(j \alpha) / j)\right)^{2} \longrightarrow d d^{c}(w-v) \wedge d d^{c} \alpha
$$

weakly. Combining this with (6) and (7) we conclude

$$
\begin{aligned}
\left(d d^{c} w\right)^{2} & =\left(d d^{c} v\right)^{2}+2 d d^{c}(w-v) \wedge d d^{c} v+d d^{c}(w-v) \wedge d d^{c}(u-v) \\
& =d d^{c} w \wedge\left(d d^{c} u+d d^{c} v\right)-d d^{c} u \wedge d d^{c} v
\end{aligned}
$$

which completes the proof of Theorem 4 .

From Theorem 4 we can immediately get the following two consequences:

Corollary 5. If $u$ is locally bounded, plurisubharmonic and $h$ is pluriharmonic, then

$$
\left(d d^{c} \max \{u, h\}\right)^{p}=d d^{c} \max \{u, h\} \wedge\left(d d^{c} u\right)^{p-1} .
$$

Corollary 6. Suppose $u, v$ are locally bounded plurisubharmonic functions with $\left(d d^{c} u\right)^{p}=0$ and $\left(d d^{c} v\right)^{q}=0$, where $1 \leq p, q \leq n$ and $p+q \leq n$. Then $\left(d d^{c} \max \{u, v\}\right)^{p+q}=0$.

The main part of the proof of (2) will be contained in

Theorem 7. Let $D$ be open in $\mathbb{C}^{n}$ and $G$ open in $\mathbb{C}^{m}$. Assume that $u, v$ are nonnegative plurisubharmonic functions in $D$ and $G$, respectively, such that

$$
\int_{\{u>0\}}\left(d d^{c} u\right)^{n}=0 \text { and } \int_{\{v>0\}}\left(d d^{c} v\right)^{m}=0 .
$$

Then, treating $u, v$ as functions on $D \times G$, we have

$$
\left(d d^{c} \max \{u, v\}\right)^{n+m}=\left(d d^{c} u\right)^{n} \wedge\left(d d^{c} v\right)^{m} .
$$


Proof. Let $w, \chi$ and $\psi_{j}$ be defined in the same way as in the proof of Theorem 4 . By Theorem 4 and since $\left(d d^{c} u\right)^{n+1}=0,\left(d d^{c} v\right)^{m+1}=0$, we have

$$
\begin{gathered}
\left(d d^{c} w\right)^{n+m}=d d^{c} w \wedge \\
{\left[\left(d d^{c} u\right)^{n-1} \wedge\left(d d^{c} v\right)^{m}+\left(d d^{c} u\right)^{n} \wedge\left(d d^{c} v\right)^{m-1}\right]} \\
-\left(d d^{c} u\right)^{n} \wedge\left(d d^{c} v\right)^{m} .
\end{gathered}
$$

Using the hypothesis on $u, v$ we may compute

$$
\begin{aligned}
d d^{c} \psi_{j} \wedge\left(d d^{c} u\right)^{n-1} & \wedge\left(d d^{c} v\right)^{m} \\
& =\left[\chi^{\prime}(0)\left(d d^{c} u\right)^{n}+j \chi^{\prime \prime}(j u) d u \wedge d^{c} u \wedge\left(d d^{c} u\right)^{n-1}\right] \wedge\left(d d^{c} v\right)^{m} \\
& =d d^{c}(\chi(j u) / j) \wedge\left(d d^{c} u\right)^{n-1} \wedge\left(d d^{c} v\right)^{m} .
\end{aligned}
$$

Since $\chi(j u) / j \downarrow u$ as $j \uparrow \infty$, it follows that

$$
d d^{c} w \wedge\left(d d^{c} u\right)^{n-1} \wedge\left(d d^{c} v\right)^{m}=\left(d d^{c} u\right)^{n} \wedge\left(d d^{c} v\right)^{m}
$$

and, similarly,

$$
d d^{c} w \wedge\left(d d^{c} u\right)^{n} \wedge\left(d d^{c} v\right)^{m-1}=\left(d d^{c} u\right)^{n} \wedge\left(d d^{c} v\right)^{m} .
$$

This, together with (8), finishes the proof.

For the proof of Theorem 1 we need a lemma which is an extension of a result from [Sa].

Lemma 8. Let $E, F, D, G$ be as in Theorem 2. For $\varepsilon>0$ set

$$
\begin{gathered}
E_{\varepsilon}:=\left\{V_{E}^{*}<\varepsilon\right\}, F_{\varepsilon}:=\left\{V_{F}^{*}<\varepsilon\right\}, \\
\widetilde{E}_{\varepsilon}:=\left\{u_{E, D}^{*}<-1+\varepsilon\right\}, \widetilde{F}_{\varepsilon}:=\left\{u_{F, G}^{*}<-1+\varepsilon\right\} .
\end{gathered}
$$

Then

$$
\begin{gathered}
V_{E_{\varepsilon}}^{*} \uparrow V_{E}^{*}, V_{F_{\varepsilon}}^{*} \uparrow V_{F}^{*}, V_{E_{\varepsilon} \times F_{\varepsilon}}^{*} \uparrow V_{E \times F}^{*}, \\
u_{\widetilde{E}_{\varepsilon}, D}^{*} \uparrow u_{E, D}^{*}, u_{\widetilde{F}_{\varepsilon}, G}^{*} \uparrow u_{F, G}^{*}, u_{\widetilde{E}_{\varepsilon} \times \widetilde{F}_{\varepsilon}, D \times G}^{*} \uparrow u_{E \times F, D \times G}^{*},
\end{gathered}
$$

as $\varepsilon \downarrow 0$, and every convergence is uniform.

Proof. The set $E \backslash E_{\varepsilon}=E \cap\left\{V_{E}^{*} \geq \varepsilon\right\}$ is pluripolar by Bedford-Taylor's theorem on negligible sets (see [BT1]). It follows that

$$
V_{E}^{*}-\varepsilon \leq V_{E_{\varepsilon}}=V_{E_{\varepsilon}}^{*} \leq V_{E}^{*}
$$

which gives the first two convergences of (9). In order to show the third one, observe that

$$
\max \left\{V_{E}, V_{F}\right\} \leq V_{E \times F} \leq V_{E}+V_{F} .
$$

Indeed, the first inequality in (11) follows easily from the definition of extremal function. Fixing one of the variables $(z, w) \in \mathbb{C}^{n} \times \mathbb{C}^{m}$, we see that the second inequality in (11) is satisfied, first on the cross $\left(E \times \mathbb{C}^{m}\right) \cup\left(\mathbb{C}^{n} \times F\right)$, and then everywhere.

By (11) $V_{E \times F} \leq 2 \varepsilon$ on $E_{\varepsilon} \times F_{\varepsilon}$. On the other hand, by (11) the set $(E \times F) \backslash$ $\left(E_{\varepsilon} \times F_{\varepsilon}\right)$ is contained in $(E \times F) \cap\left\{V_{E \times F}^{*} \geq \varepsilon\right\}$ and is thus pluripolar. Therefore

$$
V_{E \times F}^{*}-2 \varepsilon \leq V_{E_{\varepsilon} \times F_{\varepsilon}}=V_{E_{\varepsilon} \times F_{\varepsilon}}^{*} \leq V_{E \times F}^{*}
$$

and this gives (9). 
Similarly as in (11) we can show

$$
\max \left\{u_{E, D}, u_{F, G}\right\} \leq u_{E \times F, D \times G} \leq-u_{E, D} u_{F, G} .
$$

Now the proof of (10) is parallel to that of (9).

Proof of Theorem 1. If $E, F$ are compact and $L$-regular (that is, $V_{E}$ and $V_{F}$ are continuous), then (1) was shown in [Si] and (2) follows immediately from Theorem 7. For $E, F$ open we can find sequences of compact, $L$-regular sets with $E_{j} \uparrow E$ and $F_{j} \uparrow F$. Then $V_{E_{j}} \downarrow V_{E}, V_{F_{j}} \downarrow V_{F}$ and $V_{E_{j} \times F_{j}} \downarrow V_{E \times F}$ as $j \uparrow \infty$. This gives (1) and (2) for open sets. The general case can now be deduced from Lemma 8.

Proof of Theorem 2. The proof of (3) for open subsets can be found in EP. Now the proof is the same as the proof of Theorem 1.

Remark. Although (3) is stated in EP] for arbitrary subsets $E, F$, the way from open subsets to the general case is not so straightforward as the authors claim - one needs Lemma 8.

\section{REFERENCES}

[BT1] E. Bedford and B.A. Taylor, A new capacity for plurisubharmonic functions, Acta Math. 149 (1982), 1-41. MR 84d:32024

[BT2] E. Bedford and B.A. Taylor, The complex equilibrium measure of a symmetric convex set in $\mathbb{R}^{n}$, Trans. Amer. Math. Soc. 294 (1986), 705-717. MR 87f:32039

[EP] A. Edigarian and E. Poletsky, Product property of the relative extremal function, Bull. Polish Acad. Sciences 45 (1997), 331-335. MR 98i:32017

[Kl] M. Klimek, Pluripotential Theory, Clarendon Press, 1991. MR 93h:32021

[Sa] A.Sadullaev, Plurisubharmonic measures and capacities on complex manifolds, Russian Math. Surveys 36 (1981), 61-119.

[Si] J.Siciak, Extremal plurisubharmonic functions in $\mathbb{C}^{n}$, Ann. Pol. Math. 39 (1981), 175-211. MR 83e:32018

[Ze] A. Zeriahi, Fonction de Green pluricomplexe à pôle à l'infini sur un espace de Stein parabolique et applications, Math. Scand. 69 (1991), 89-126. MR 93a:32031

Institute of Mathematics, Jagiellonian University, Reymonta 4, 30-059 Kraków, POLAND

E-mail address: blocki@im.uj.edu.pl 